\title{
Cervical gunshot wound: bullet trajectory on three-dimensional computed tomography scan
}

\author{
Ferimento por arma de fogo em região cervical: trajetória da bala na tomografia \\ computadorizada tri-dimensional \\ Juliana Cabrera Garrido', Frederico Figueiredo Amâncio², Rodrigo Moreira Faleiro³, Geraldo Magela \\ Ribeiro4 ${ }^{4}$ Thiago Cardoso Vale ${ }^{5}$
}

An 18-year-old man was admitted to the Emergency Unit shortly after having a gunshot wound in the neck. The patient developed an acute flaccid tetraplegia combined with arreflexia and sphincter impairment. He was promptly intubated and immobilized with a cervical spine collar. Threedimensional (3D) cervical computed tomography (CT) scan showed the bullet trajectory with entrance on the right side and no exit on the left side (Figure). A C4-C5 bone fracture was observed, as well as right jugular vein thrombosis. All the greater cervical arteries were incredibly spared. Threedimensional CT scan imaging techniques can be a useful tool for evaluating gunshot wounds of the cervical region ${ }^{1}$.
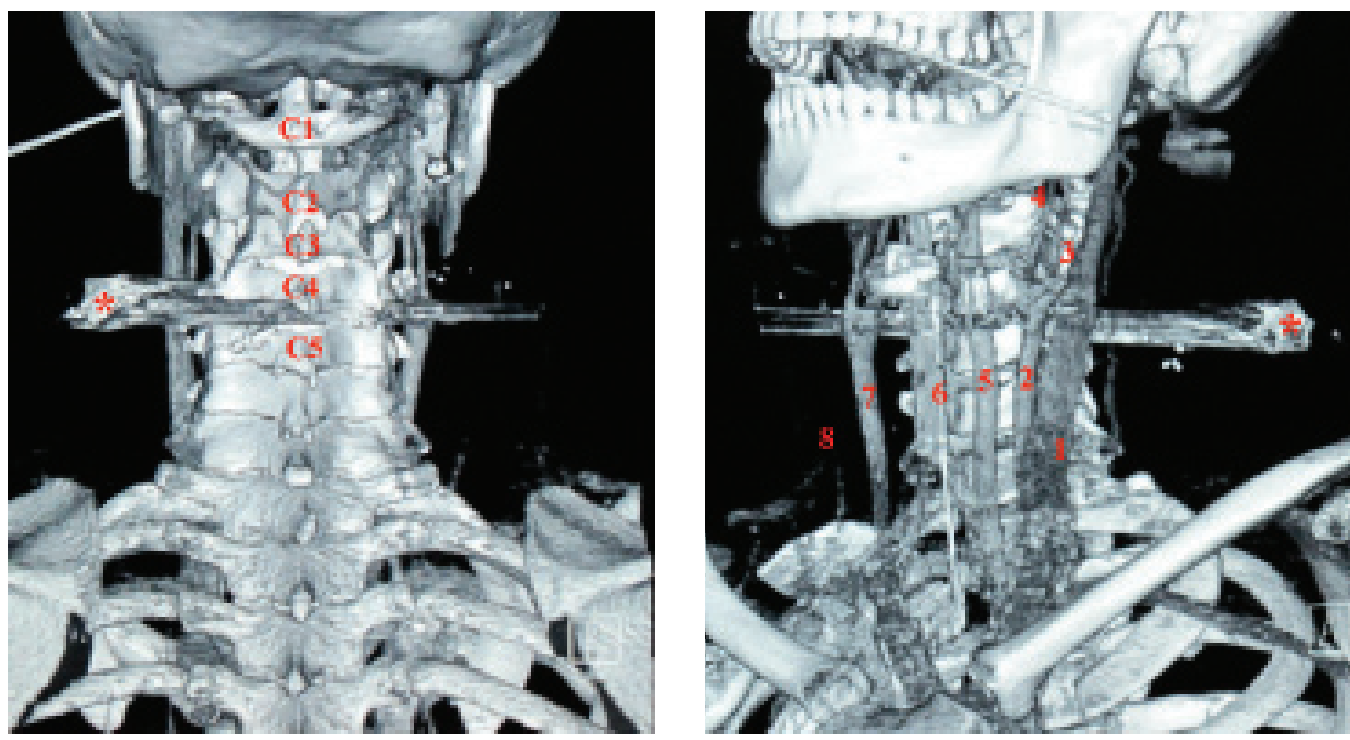

Figure. (1) left jugular vein, (2) left common carotid artery, (3) left external carotid artery, (4) left internal carotid artery, (5) nasogastric tube, (6) endotracheal tube, (7) right common carotid artery, (8) thrombosed right jugular vein.

1. Bruner D, Gustafson CG, Visintainer C. Ballistic injuries in the

emergency department. Emerg Med Pract 2011;13:1-30.

\footnotetext{
'MD, Internal Medicine Resident at Hospital João XXIII, Fundação Hospitalar do Estado de Minas Gerais, Belo Horizonte MG, Brazil; ${ }^{2} \mathrm{MD}$, Intensivist at Hospital João XXIII, Fundação Hospitalar do Estado de Minas Gerais, Belo Horizonte MG, Brazil;

${ }^{3} \mathrm{MD}$, Neurosurgeon, Head of the Residency Program of Neurosurgery at Hospital João XXIII, Fundação Hospitalar do Estado de Minas Gerais, Belo Horizonte MG, Brazil;

${ }^{4}$ MD, Radiologist at Hospital João XXIII, Fundação Hospitalar do Estado de Minas Gerais, Belo Horizonte MG, Brazil;

${ }^{5} \mathrm{MD}$, Neurologist at Hospital das Clínicas, Universidade Federal de Minas Gerais, Belo Horizonte MG, Brasil.

Correspondence: Frederico Figueiredo Amâncio; Hospital João XXIII, Fundação Hospitalar do Estado de Minas Gerais; Avenida Professor Alfredo Balena, 400; 30130-100 Belo Horizonte MG - Brasil; E-mail:manzoff@gmail.com

Conflict of interest: There is no conflict of interest to declare.

Received 27 February 2012; Received in final form 11 June 2012; Accepted 28 June 2012
} 